\title{
ON NON-DENUMERABLE GRAPHS
}

\author{
P. ERDÖS AND S. KAKUTANI
}

The present paper consists of two parts. In Part 1 we prove a theorem on the decomposition of a complete graph. This result is then applied in Part 2 to show that the continuum hypothesis is equivalent to the possibility of decomposing the set of all real numbers into a countable number of summands each consisting of rationally independent numbers.

\section{PART 1}

A graph $G$ is complete if every pair of points of $G$ is connected by one and only one segment. $G$ is called a tree if it does not contain any closed polygon.

TheOREM 1. A complete graph of cardinal number $m$ (that is, the cardinal number of the vertices is $m$ ) can be split up into a countable number of trees if and only if $m \leqq \boldsymbol{N}_{1}$.

Proof. We shall first prove that every complete graph of power $\boldsymbol{\aleph}_{1}$ can be split up into the countable sum of trees. ${ }^{1}$ Let $G$ be a complete graph of cardinal number $\boldsymbol{\aleph}_{1}$. Let $\left\{x_{\alpha}\right\}, \alpha<\omega_{1}$, be any well ordered set of power $\boldsymbol{\aleph}_{1}$. We may assume that $G$ is represented by a system of segments $\left(x_{\alpha}, x_{\beta}\right), \alpha<\beta<\omega_{1}$. For any $\beta<\omega_{1}$ arrange the set of all $\alpha<\beta$ into a sequence $\alpha_{\beta, n}, n=1,2, \ldots$, and let $G_{n}$ be the set of all segments $\left(x_{\alpha}, x_{\beta}\right)$ such that $\alpha=\alpha_{\beta, n}$. It is clear that $G=\cup_{n=1}^{\infty} G_{n}$ and that for each $G_{n}$, for every $\beta<\omega_{1}$, there exists one and only one $\alpha$ such that $\left(x_{\alpha}, x_{\beta}\right) \in G_{n}$ and $\alpha<\beta$. From this last fact it is clear that $G_{n}$ does not contain any closed polygon.

Conversely, let us assume that a complete graph $G$ of cardinal number $m$ is split up into a countable number of trees $T_{n} ; G=\cup_{n=1}^{\infty} T_{n}$. We shall prove that $m \leqq \boldsymbol{N}_{1}$. We can again assume that $G$ is represented by a system of segments $\left(x_{\alpha}, x_{\beta}\right), \alpha<\beta<\phi$, where $\left\{x_{\alpha}\right\}, \alpha<\phi$, is a well ordered set of cardinal number $m$.

We shall first decompose each $T_{n}$ into four parts $T_{n, i}, i=1,2,3,4$, such that $T_{n, 1}$ and $T_{n, 2}$ satisfy the condition:

(1) Any two consecutive segments of the graphs $T_{n, 1}$ and $T_{n, 2}$ are of the form: $\left(x_{\alpha}, x_{\beta}\right),\left(x_{\alpha}, x_{\gamma}\right), \alpha<\beta, \alpha<\gamma, \beta \neq \gamma$. And $T_{n, 3}, T_{n, 4}$ satisfy:

(2) Any two consecutive segments of the graphs are of the form: $\left(x_{\beta}, x_{\alpha}\right),\left(x_{\gamma}, x_{\alpha}\right), \beta<\alpha, \gamma<\alpha, \beta \neq \gamma$.

Received by the editors September 18, 1942.

${ }^{1}$ This result was also obtained by J. Tukey, oral communication. 
For this purpose, let $T_{n}=\cup_{\lambda} T{ }_{n}^{(\lambda)}$ be a decomposition of each $T$ into its components $T_{n}^{(\lambda)}$. The number of components $T_{n}^{(\lambda)}$ is not necessarily countable. Let $x_{n}^{(\lambda)}$ be an arbitrary point in each $T_{n}^{(\lambda)}$, called the origin of $T_{n}^{(\lambda)}$. A segment $\left(x_{\alpha}, x_{\beta}\right), \alpha<\beta$, of $T_{n}^{(\lambda)}$ is of 0 th degree of the first kind if $x_{\alpha}=x_{n}^{(\lambda)}$ and is of 0th degree, of the second kind if $x_{\beta}=x_{n}^{(\lambda)}$. Assume that the segments of degree $0,1, \cdots, m-1$ of the first and second kind are already defined in $T_{n}^{(\lambda)}$. Then a segment $\left(x_{\alpha}, x_{\beta}\right)$, $\alpha<\beta$, of $T_{n}^{(\lambda)}$ is of the $m$ th degree, of the first kind if $x_{\alpha}$ is one of the end points of a segment of degree $m-1$ and if $\left(x_{\alpha}, x_{\beta}\right)$ is not of degree at most $m-1$. The segments of degree $m$ of the second kind are analogously defined. In this way it is possible to define a degree of first or second kind for every segment of $T_{n}^{(\lambda)}$. Let $T_{n, m}^{(\lambda)^{\prime}}$ be the set of all segments of $T_{n}^{(\lambda)}$ of degree $m$ of the first kind, and let $T_{n, m}^{(\lambda) \prime \prime}$ be the set of all segments of $T_{n}^{(\lambda)}$ of degree $m$ and of second kind. It is clear that $T_{n, m}^{(\lambda)^{\prime}}$ and $T_{n, m}^{(\lambda)^{\prime \prime}}$ satisfy conditions (1) and (2), respectively. If we put

$$
\begin{array}{ll}
T_{n, 1}=\cup_{\lambda} \bigcup_{2 m} T_{n, 2 m}^{(\lambda)^{\prime}}, & T_{n, 2}=\cup_{\lambda} \bigcup_{2 m+1} T_{n, 2 m+1}^{(\lambda)^{\prime}}, \\
T_{n, 3}=\cup_{\lambda} \bigcup_{2 m} T_{n, 2 m}^{(\lambda)^{\prime \prime}}, & T_{n, 4}=\cup_{\lambda} \bigcup_{2 m+1} T_{n, 2 m+1}^{(\lambda)^{\prime \prime}}
\end{array}
$$

then $T_{n}=\cup_{i=1}^{4} T_{n, i}$ is a required decomposition. The conditions (1) and (2) are satisfied by $T_{n, 1}, T_{n, 2}$ and $T_{n, 3}, T_{n, 4}$, respectively, since the summands on the right-hand side are disjoint.

Thus we have obtained a decomposition of $T$ into a countable number of parts $T_{n, i}, n=1,2 \cdots ; i=1,2,3,4$. We observe that from (1) and (2) follows (3) and (4), respectively:

(3) For any $\alpha<\phi$, there exists at most one $\beta<\alpha$ such that $\left(x_{\beta}, x_{\alpha}\right)$ belongs to the graph.

(4) For any $\alpha<\phi$ there exists at most one $\beta>\alpha, \beta<\phi$, such that $\left(x_{\alpha}, x_{\beta}\right)$ belongs to the graph.

Let us now put

$$
\begin{aligned}
T^{\prime} & =\cup_{n=1}^{\infty} T_{n, 1} \cup \cup_{n=1}^{\infty} T_{n, 2}, \\
T^{\prime \prime} & =\cup_{n=1}^{\infty} T_{n, 3} \cup \cup_{n=1}^{\infty} T_{n, 4} .
\end{aligned}
$$

Then $T^{\prime}$ and $T^{\prime \prime}$ clearly satisfy the following conditions:

(5) For any $\alpha<\phi$ the set of all $\beta<\alpha$ such that $\left(x_{\beta}, x_{\alpha}\right)$ belongs to the graph is countable.

(6) For any $\alpha<\phi$ the set of all $\beta>\alpha, \beta<\phi$ such that $\left(x_{\alpha}, x_{\beta}\right)$ belongs to the graph is countable.

From this it easily follows by the same argument as in W. Sierpinski ${ }^{2}$ that the cardinal number $m$ of all points $x_{\alpha}, \alpha<\phi$, is at most $\aleph_{1}$.

${ }^{2}$ W. Sierpinski, Hypothese $d u$ continu, Proposition $\mathrm{P}_{1}$, p. 9. 
Similarly we can prove that the complete graph of power $\boldsymbol{\aleph}_{x}$ is the sum of $\boldsymbol{\aleph}_{x-1}$ trees, but not the sum of less than $\boldsymbol{\aleph}_{x-1}$ trees. We can put the following problem: Is the complete graph of power $\boldsymbol{\aleph}_{x}$ the sum of less than $\boldsymbol{\aleph}_{x-1}$ such graphs which do not contain a quadrilateral? We cannot answer this question, unless we assume the generalized hypothesis of the continuum, in which case the answer is negative. It can be shown that the complete graph of power $2^{m}$ is the sum of $m$ graphs, which do not contain even closed polygons, ${ }^{3}$ but that the complete graph of power greater than $2^{m}$ is not the sum of $m$ graphs which do not contain triangles. ${ }^{4}$

\section{PART 2}

THEOREM 2. The continuum hypothesis is equivalent to the following proposition:

(P) The set of all real numbers can be decomposed into a countable number of subsets, each consisting only of rationally independent numbers.

Proof. We shall first prove that the continuum hypothesis implies proposition (P). Let $\xi_{\alpha}, \alpha<\omega_{1}$, be a Hamel basis for the set $R$ of all real numbers, well ordered in a transfinite sequence of type $\omega_{1}$; that is, the $\xi_{\alpha}$ are rationally independent, and every real number $x(\neq 0)$ can be uniquely expressed in the following form:

$$
x_{\alpha}=\sum_{i=1}^{n} r_{i} \xi_{\alpha_{i}}, \quad \alpha_{1}<\alpha_{2}<\cdots<\alpha_{n}<\omega_{1},
$$

where the $r_{i}$ are rational numbers different from 0 , and $n$ is a positive integer.

For any finite system of rational numbers $r_{1}, r_{2}, \cdots, r_{n}$ let $R_{r_{1}, r_{2}, \cdots, r_{n}}$ be the set of all $x \in R$ which are expressed in the form (2.1). Then

$$
R=(0) \cup \bigcup_{\left(r_{1}, r_{2}, \cdots, r_{n}\right)} R_{r_{1}, r_{2}}, \cdots, r_{n}
$$

is a decomposition of the set $R$ into the set (0) consisting of 0 alone and a countable number of sets $R_{r_{1}, r_{2}, \ldots, r_{n}}^{(\alpha)}$, where $U_{\left(r_{1}, r_{2}, \ldots, r_{n}\right)}$ means the union for all possible ordered systems $r_{1}, r_{2}, \cdots, r_{n}$ of rational numbers. Consequently in order to prove our theorem it suffices to prove it for all the $R_{r_{1}, r_{2}, \cdots, r_{n}}$.

For each ordinal number $\alpha, \alpha<\omega_{1}$, let $R_{r_{1}, r_{2}}^{(\alpha)}, \ldots, r_{n}$ be the subset of $R_{r_{1}, r_{2}}, \cdots, r_{n}$ consisting of all real numbers $x$, such that $\alpha_{n}=\alpha_{n}(x)=\alpha$.

${ }^{3} \mathrm{~K}$. Gödel, oral communication.

${ }^{4} \mathrm{P}$. Erdös, On graphs and nets, to appear in Revista, Matematicas y Fisica Teorica. 
Then since $\alpha_{1}<\alpha_{2}<\cdots<\alpha_{n}=\alpha$, each $R_{r_{1}, r_{2}, \cdots, r_{n}}^{(\alpha)}$ is a countable set. Let us arrange the set $R_{r_{1}, r_{2}, \cdots, r_{n}}^{(\alpha)}$ in to a sequence $\left\{x_{r_{1}, r_{2}, \cdots, r_{n}, m}^{(\alpha)}\right\}$, $m=1,2, \cdots$, and let us put $S_{r_{1}, r_{2}, \cdots, r_{n}, m}=\left\{x_{r_{1}, r_{2}}^{(\alpha)}, \ldots, r_{n}, m, \alpha<\omega_{1}\right\}$. Clearly $\alpha_{n}(x) \neq \alpha_{n}(y)$ for any $x, y \in S_{r_{1}, r_{2}}, \cdots, r_{n}, m, x \neq y$, and $R_{r_{1}, r_{2}}, \cdots, r_{n}$ $=\bigcup_{m=1}^{\infty} S_{r_{1}, r_{2}}, \cdots, r_{n}, m$.

We shall prove that each $S_{r_{1}, r_{2}}, \cdots, r_{n}, m$ consists only of rationally independent numbers. In fact, if we have

$$
\sum_{i=1}^{k} p_{i} x_{i}=0
$$

where $x_{i} \in S_{r_{1}, r_{2}, \cdots, r_{n}, m}, i=1, \cdots, k ; x_{i} \neq x_{j}(i=j)$, and $p_{i}$ is an integer different from 0 , for $i=1, \cdots, k$, then there would exist an integer $i_{0}$ for which $\alpha^{*}=\alpha_{n}\left(x_{i_{0}}\right)>\alpha_{n}\left(x_{i}\right)$ for all $i \neq i_{0}$. Hence, in the expansion (2.1) of all the $x_{i}, i=1, \cdots, n$, the term $\xi_{\alpha^{*}}$ would appear only once, which is clearly impossible because of (2.3). Thus we have proved that the continuum hypothesis implies the proposition $(\mathrm{P})$.

Conversely, let us assume that the proposition $(\mathrm{P})$ is true. We shall prove the continuum hypothesis from it. We shall prove this by showing that, under the assumption $(\mathrm{P})$ the complete graph of power $2^{\mathrm{N}_{0}}$ is the sum of a countable number of trees (see Theorem 1).

Let $R=\cup_{n=1}^{\infty} M_{n}$ be a decomposition of the set of all real numbers in to a countable sum of sets each consisting of rationally independent numbers. At least one of the $M_{n}$ must have power $2^{N_{0}}$. (This follows from a well known theorem of J. König. ${ }^{5}$ ) We may assume that $M_{1}$ has power $2^{\boldsymbol{N}_{0}}$. Let $G$ be the complete graph of cardinal number $2^{\boldsymbol{N}_{0}}$. We may assume that $G$ is represented by a system of segments $(x, y)$, $x, y \in M_{1}, x<y$. Let $G_{n}$ be a subgraph of $G$, consisting of all those segments $(x, y), x<y$ for which $y-x \in M_{n}$. Clearly $G=\cup_{n=1}^{\infty} G_{n}$. We shall prove that each $G_{n}$ is a tree. In fact assume that $G_{n}$ contains a closed polygon. Let $x_{1}, \cdots, x_{k}, x_{k+1}=x_{1}$ be the vertices of this polygon. Then $0=\sum_{i=1}^{k}\left(x_{i}-x_{i+1}\right)=\sum_{i=1}^{k} \pm\left|x_{i}-x_{i+1}\right|$. On the other hand $\left|x_{i}-x_{i+1}\right| \in M_{n}$ by construction, and since all $x_{i_{r}} \in M_{1}$ the numbers $\left|x_{i}-x_{i+1}\right|, i=1,2, \cdots, k$, are all different. This is however a contradiction since $M_{n}$ consists of rationally independent numbers. This completes the proof of Theorem 2 .

It seems likely that the following stronger theorem also holds: Assume that the continuum hypothesis is false, and let the sets $M_{n}$ consist of rationally independent numbers. Then $\cup_{n=1}^{\infty} M_{n}$ has inner measure 0 .

5 J. König, see W. Sierpinski, ibid. p. 6. 
We can of course prove the following theorem: The necessary and sufficient condition for the continuum to be of power $\boldsymbol{\aleph}_{x+1}$ is that $R$ shall be the sum of $\boldsymbol{\aleph}_{x}$ sets consisting of rationally independent numbers, and that $R$ shall not be the sum of less than $\boldsymbol{\aleph}_{x}$ such sets. The proof is the same as that of Theorem 2.

University of Pennsylvania and

The Institute for Advanced Study

\section{ADDENDUM TO THE PAPER "GENERALIZED FISCHER GROUPS AND ALGEBRAS"}

\section{R. H. BRUCK}

The author regrets the omission, in a paper which recently appeared, ${ }^{1}$ of an important reference to a paper by N. Jacobson. ${ }^{2}$ Indeed Lemma IIIa of the author's paper, and its immediate consequence, Theorem I, are rather special cases (albeit independently obtained) of Theorem I of the latter paper. Accordingly Professor Jacobson's name should have appeared in the introduction along with those of M. Schiffer and W. Specht, and chronologically before that of Specht.

UNIVERSITY OF WISCONSIN

Received by the editors December 9, 1942.

1 R. H. Bruck, Generalized Fischer groups and algebras, Bull. Amer. Math. Soc. vol. 48 (1942) pp. 618-626, in particular p. 623.

2 N. Jacobson, Normal semi-linear transformations, Amer. J. Math. vol. 61 (1939) pp. $45-58$, in particular p. 49. 